\title{
Docosahexaenoic Acid
}

National Cancer Institute

\section{Source}

National Cancer Institute. Docosahexaenoic Acid. NCI Thesaurus. Code C68345.

A polyunsaturated very long-chain fatty acid with a 22-carbon backbone and 6 double bonds. Four separate isomers can be called by this name. 